\title{
Trace N-glycans including sulphated species may originate from various plasma glycoproteins and not necessarily IgG
}

\author{
Gordan Lauc (10 ${ }^{1,2}$, Frano Vučković ${ }^{2}$, Albert Bondt (1D ${ }^{3}$, Marija Pezer (1D ${ }^{2}$ \& Manfred Wuhrer (D) ${ }^{3}$
}

In the article entitled "A method to identify trace sulphated IgG $\mathrm{N}$-glycans as biomarkers for rheumatoid arthritis", Wang et al. reported an analysis of glycans in 277 patients with rheumatoid arthritis (RA) and 141 healthy individuals ${ }^{1}$. The analytical method they used was very sensitive and powerful, providing results for glycans that represented only $0.005 \%$ of the glycome. They identified a total of 444 glycan structures and concluded that all these structures are $\mathrm{N}$-glycans that are attached to immunoglobulin $\mathrm{G}(\mathrm{IgG})$. However, this glycomic analysis of IgG obtained by protein A purification does not account for the ubiquitous presence of varying levels of other contaminating plasma glycoproteins. As experimental evidence for association of these trace glycans with IgG was not provided, we believe that many of these glycans actually originated from other plasma glycoproteins, and not IgG.

In the past few years we have analysed the glycome composition of more than 50,000 samples from different clinical and epidemiological studies ${ }^{2-13}$. The quality assurance measures we took enabled us to reliably determine sources of experimental variation and levels of experimental error in glycomic experiments. From these analyses we learned that one of the major sources of error in IgG glycome analysis is linked to glycoprotein contaminants. Affinity purification of IgG using proteins A or G is never absolute and varying levels of contaminating plasma proteins are always present. As most plasma proteins are glycosylated, variations in levels of contaminating proteins can have substantial effect on the apparent IgG glycome composition. For example, the diantennary, digalactosylated and disialylated glycan without core fucose (H5N4S2, or A2G2S2 in alternative nomenclature) is the most prevalent glycan on total plasma proteins, while it is present in very low amounts in the total IgG glycome (Fig. 1a-c), and practically nonexistent on IgG Fc 3 ,11,12.

When released IgG glycans are analysed, even with very low levels of contaminating plasma proteins (not even visible on Coomassie-stained SDS-PAGE), the majority of H5N4S2 glycans in the "IgG glycome" actually originate from other plasma proteins such as transferrin, and not $\operatorname{IgG}^{14}$. Wang et al. ${ }^{1}$ reported that, in their study, two H5N4S2 isomers had a combined relative abundance of $5.4 \%$ (Supplementary Table 4). In our highthroughput studies of released IgG glycans (on over 30,000 individuals) this structure was less than $1 \%$ in nearly all samples. Both ultra-high performance liquid chromatography (UHPLC) and matrix-assisted laser desorption/ionization-time of flightmass spectrometry (MALDI-TOF-MS) analyses resulted in comparable levels of this glycan (Fig. 1b, c) ${ }^{6,7,15}$. This structure was slightly elevated in patients with RA, mostly originating from glycans attached to Fab, but still below $1.5 \%$ of the total IgG glycome (Fig. 1c).

We consider that high levels of H5N4S2 are an indicator of contamination with other glycoproteins and use it for quality assessment of IgG purification. The fact that the H5N4S2 glycan represents over $5 \%$ of the IgG glycome in the study of Wang et al. ${ }^{1}$ indicates that the purified IgG may contain substantial levels of contaminating plasma glycoproteins. Furthermore, this glycan had a particularly high standard deviation, again suggesting that it was mainly derived from varying levels of contaminating plasma glycoproteins. The study by Wang et al. ${ }^{1}$ may therefore be confounded by sample purity issues, and the claim that the novel glycans are linked to IgG seems insufficiently supported. To the contrary, many of these low-abundance glycan structures may be derived from contaminating plasma proteins, and not or only to a lesser extent from IgG.

Figure 3 of Wang et al. ${ }^{1}$ implies that the novel glycan structures are actually $\mathrm{Fc}$ glycans, which may be questioned as the human IgG glycome is composed of glycans from the $\mathrm{Fc}$ as well as the Fab portion of the molecule. A state-of-the-art method of confirming IgG as the carrier molecule of the glycans and revealing the attachment site in the Fc portion of the molecule would be a complementary glycopeptide analysis. In the study by Wang et al. ${ }^{1}$ this was not performed and therefore the claim that $\operatorname{IgG}$ is the carrier of the 444 different $\mathrm{N}$-glycan structures is not substantiated with experimental evidence.

We do not imply that any of the experimental data presented in the manuscript are false, or that association with rheumatoid

\footnotetext{
${ }^{1}$ Faculty of Pharmacy and Biochemistry, University of Zagreb, Ante Kovacica 1, 10000 Zagreb, Croatia. ${ }^{2}$ Genos Glycoscience Research Laboratory, Borongajska 83H, 10000 Zagreb, Croatia. ${ }^{3}$ Center for Proteomics and Metabolomics, Leiden University Medical Centre, Zone S3, Albinusdreef 2, 2333 ZA Leiden, The Netherlands. Correspondence and requests for materials should be addressed to G.L. (email: glauc@pharma.hr)
} 
a
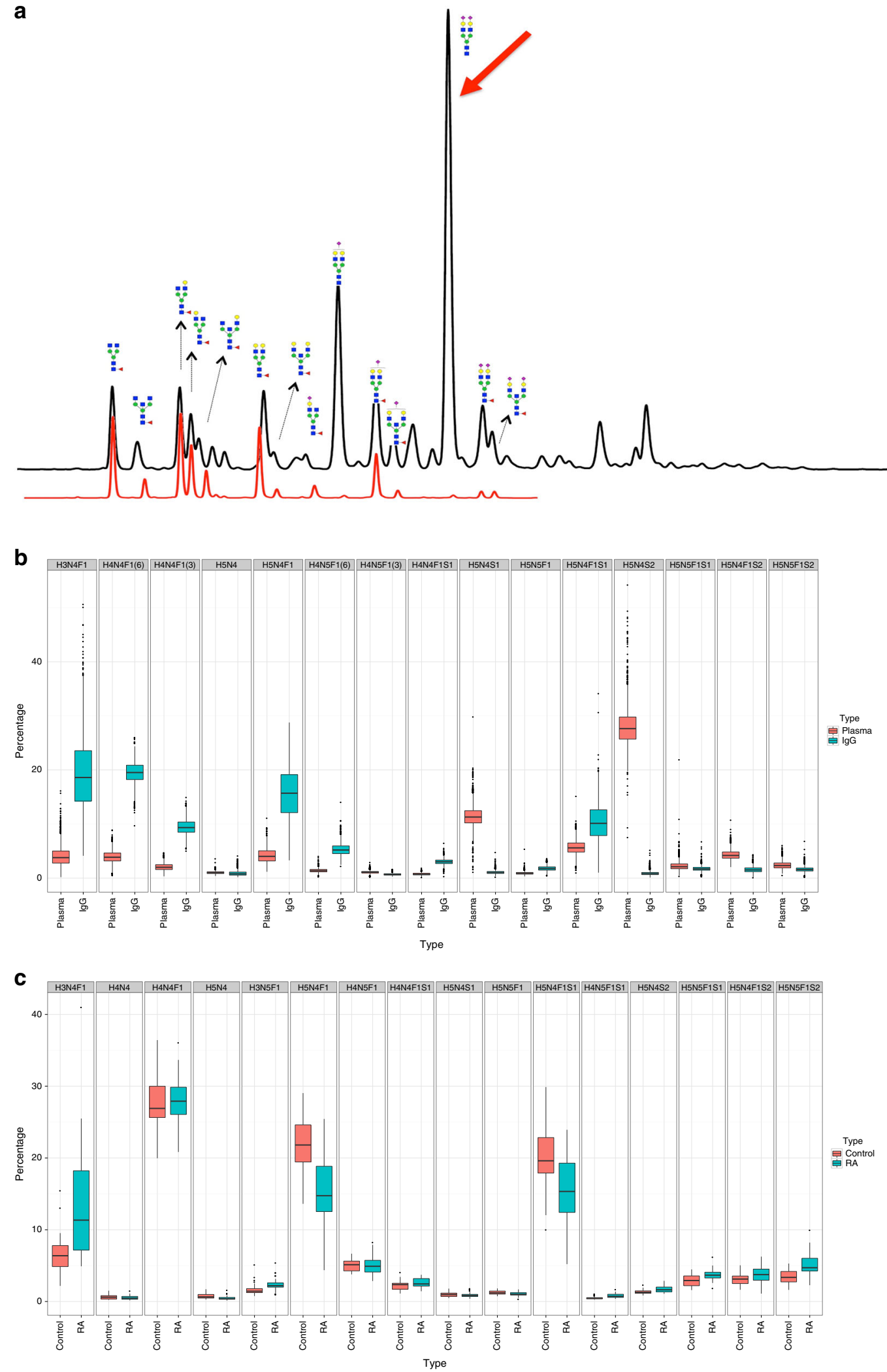

Fig. 1 lgG and total plasma N-glycomes. a An overlay of UHPLC traces of IgG (red) and total plasma (black) glycomes. Major structures that are present in both total plasma and lgG glycomes are presented as cartoons composed of $\mathrm{N}$-acetylglucosamine (blue square), mannose (green circle), galactose (yellow circle), fucose (red triangle) and sialic acid (purple diamond). The red arrow marks the largest plasma glycan peak that contains the diantennary, digalactosylated and disialylated glycan without core fucose (H5N4S2). This structure is present only in trace amounts in the total lgG glycome pool and is completely undetectable as a corresponding Fc glycopeptide ${ }^{3,11,12}$. b Composition of the total plasma and IgG N-glycomes in 2611 individuals from the general population analysed by UHPLC 5 . c Comparison of major lgG glycan structures in 31 patients with RA and 25 age-matched healthy individuals measured by MALDI-TOF-MS 15 
arthritis is unsubstantiated. However, we challenge the conclusion that the analysed glycans originate from IgG, as this claim is not substantiated by the experimental evidence.

\section{Methods}

UHPLC analysis of IgG and total plasma N-glycome was performed on 2611 individuals as described previously ${ }^{5,6}$. Briefly, IgG was isolated by affinity chromatography on protein G. N-glycans from isolated IgG and from plasma samples were released with PNGase F, labelled with 2-aminobenzamide and analysed by UHPLC. IgG N-glycan chromatograms were separated into 24 and total plasma Nglycan chromatograms into 39 glycan peaks. The content of each glycan peak had been determined previously by MS and exoglycosidase digestion.

MS analysis of IgG N-glycome in 31 patients with RA and 25 age-matched healthy individuals was performed as described previously ${ }^{15}$. Briefly, IgG was isolated by affinity chromatography on CaptureSelect IgG-Fc (Hu) beads. Nglycans from isolated IgG were released with PNGase F, ethyl esterification was performed to prevent the loss of sialic acids, and released glycans were analysed by MALDI-TOF-MS

Data availability. All relevant data are available from the corresponding author upon reasonable request.

Received: 7 October 2017 Accepted: 14 June 2018

Published online: 25 July 2018

\section{References}

1. Wang, J. R. et al. A method to identify trace sulfated IgG N-glycans as biomarkers for rheumatoid arthritis. Nat. Commun. 8, 631 (2017).

2. Barrios, C. et al. Glycosylation profile of IgG in moderate kidney dysfunction. J. Am. Soc. Nephrol. 27, 933-941 (2016).

3. Huffman, J. E. et al. Comparative performance of four methods for highthroughput glycosylation analysis of immunoglobulin G in genetic and epidemiological research. Mol. Cell. Proteom. 13, 1598-1610 (2014).

4. Keser, T. et al. Increased plasma N-glycome complexity is associated with higher risk of type 2 diabetes. Diabetologia 60, 2352-2360 (2017).

5. Kristic, J. et al. Glycans are a novel biomarker of chronological and biological ages. J. Gerontol. 69, 779-789 (2014).

6. Novokmet, M. et al. Changes in IgG and total plasma protein glycomes in acute systemic inflammation. Sci. Rep. 4, 4347 (2014).

7. Shen, X. et al. Multivariate discovery and replication of five novel loci associated with Immunoglobulin G N-glycosylation. Nat. Commun. 8, 447 (2017).

8. Vučković, F. et al. Systemic lupus erythematosus associates with the decreased immunosuppressive potential of the IgG glycome. Arthritis Rheumatol. 67, 2978-2989 (2015).

9. Vučković, F. et al. IgG glycome in colorectal cancer. Clin. Cancer Res. 22, 3078-3086 (2016)
10. Epp, A. et al. Sialylation of IgG antibodies inhibits IgG-mediated allergic reactions. J. Allergy Clin. Immunol. 141, 399-402.e8 (2018).

11. Kapur, R. et al. Low anti-RhD IgG-Fc-fucosylation in pregnancy: a new variable predicting severity in haemolytic disease of the fetus and newborn. $\mathrm{Br}$. J. Haematol. 166, 936-945 (2014).

12. Kemna, M. J. et al. Galactosylation and sialylation levels of IgG predict relapse in patients with PR3-ANCA associated vasculitis. EBioMedicine 17, 108-118 (2017).

13. Simurina, M. et al. Glycosylation of immunoglobulin $\mathrm{G}$ associates with clinical features of inflammatory bowel diseases. Gastroenterology 154, 1320-1333. e1310 (2018).

14. Clerc, F. et al. Human plasma protein N-glycosylation. Glycoconj. J. 33, 309-343 (2016).

15. Bondt, A., Wuhrer, M., Kuijper, T. M., Hazes, J. M., \& Dolhain, R. J. Fab glycosylation of immunoglobulin $\mathrm{G}$ does not associate with improvement of rheumatoid arthritis during pregnancy. Arthritis Res. Ther. 18, 274 (2016).

\section{Author contributions}

G.L. and M.W. conceived the paper; G.L., M.W., A.B., and M.P. wrote the paper; A.B. and F.V. performed the data analysis. All authors read and approved the final manuscript.

\section{Additional information}

Competing interests: G.L. is the founder and CEO of Genos Ltd, a private research organization that specializes in high-throughput glycomic analysis and has several patents in this field. F.V. and M.P. are employees of Genos Ltd. The remaining authors declare no competing interests.

Reprints and permission information is available online at http://npg.nature.com/ reprintsandpermissions/

Publisher's note: Springer Nature remains neutral with regard to jurisdictional claims in published maps and institutional affiliations.

pen Access This article is licensed under a Creative Commons Attribution 4.0 International License, which permits use, sharing, adaptation, distribution and reproduction in any medium or format, as long as you give appropriate credit to the original author(s) and the source, provide a link to the Creative Commons license, and indicate if changes were made. The images or other third party material in this article are included in the article's Creative Commons license, unless indicated otherwise in a credit line to the material. If material is not included in the article's Creative Commons license and your intended use is not permitted by statutory regulation or exceeds the permitted use, you will need to obtain permission directly from the copyright holder. To view a copy of this license, visit http://creativecommons.org/ licenses/by/4.0\%

(C) The Author(s) 2018 